\section{Response times to different spatial frequencies: Is there a $100-$ msec rule?}

\section{ALISON BOWLING and WILLIAM LOVEGROVE University of Tasmania, \\ P.O. Box 252C, Hobart, Australia 7005}

Stanley, Howell, and Smith (1980) present an alternative analysis of the persistence data obtained by Bowling and Lovegrove (1980). They argue that stimulus onset asynchrony (SOA) is a more appropriate measure of persistence than interstimulus interval (ISI). In addition, they propose that the use of this measure alters the conclusion made by Bowling and Lovegrove (1980) that the stimulus duration at which persistence ceases to decline steeply with increasing stimulus duration increases with spatial frequency and is related to temporal integration. We consider that SOA is a measure of the total duration of the response to a stimulus, rather than of persistence, and use of this measure does not affect the major conclusions of Bowling and Lovegrove (1980).

Stanley et al. (1980) also mentioned that a nonlinear continuous function may provide a better fit to Bowling and Lovegrove's data than a two-limb linear function. This possibility was evaluated by fitting linear, quadratic, and logarithmic functions to the data by the method of least squares. The sums of the squared deviations of the data points from each of these regression relationships and from the two-limb linear relationship were calculated. Table 1 shows that, for each spatial frequency, the deviation of the data points from the two-component linear relationship was lower than that for any other relationship. The two-limb relationship is therefore a better fit to the data than any of the other possibilities investigated.

The question as to whether SOA or ISI is the more appropriate measure will be considered in terms of the temporal property measured by these. Using arrays of small light flashes, Di Lollo $(1977,1980)$ has provided data indicating that the response to a brief stimulus commences at onset and continues for approximately $100 \mathrm{msec}$ (the 100 -msec rule?). That is, the total duration of the response produced by a brief stimuli is approximately constant. In the measurement technique employed by Bowling and Lovegrove (1980), the SOA is consequently a measure of this total response duration (and of the duration of any persistence occurring apart from this). That part of the response that occurs during the actual presence of the stimulus cannot be considered to be persistence, however, since, by definition, persistence is a continued visible representation of the stimulus occurring after its offset. Persistence is consequently the difference between SOA and stimulus durationthe ISI.

Di Lollo's data show that the total response duration remains approximately constant at about $100 \mathrm{msec}$. Consequently, the persistence durations of stimuli shorter than this decline linearly with increasing stimulus duration with a slope of -1.0 , a result also obtained by Efron (1970). This approximately constant response duration may be a further manifestation of the limited temporal resolution of the visual system, of which the best known example is Bloch's law. Under conditions of perfect temporal integration, the response duration of stimuli shorter than the critical duration would be invariant with stimulu duration. There are, however, some cases in which perfect reciprocity does not occur at threshold. These include large field size (Owen, 1972) and patterned stimuli (Breitmeyer \& Ganz, 1977; Legge, 1978). Under these conditions, the total response duration may increase slightly with increasing stimulus duration up to the critical duration. In this case, the slope of the initial portion of the relationship between persistence and stimulus duration would be more shallow than -1.0 , as Bowling and Lovegrove (1980) observed.

Stanley et al. (1980) have argued that use of SOA as the dependent variable produced an approximately constant relationship between SOA and stimulus duration at brief stimulus duration for each spatial frequency used. This is not surprising, in view of the relationship between persistence and stimulus duration discussed above. The SOA obtained with brief stimuli did, however, increase slightly with increasing stimulus duration, reflecting the fact that the initial slope of the persistence by duration relationship was not -1.0 . Stanley et al. (1980) argue that their reanalysis alters the conclusion of Bowling and Lovegrove (1980) that the duration at which a change in the slope of this relationship occurs is dependent upon spatial frequency, and consequently, that one component is a manifestation of the same processes underlying temporal integration at threshold. They

Table 1

Sums of the Squared Deviations of the Data of Bowling and Lovegrove (1980) From Various Possible Regression Relationships

\begin{tabular}{lrrr} 
& \multicolumn{3}{c}{$\begin{array}{c}\text { Spatial Frequency } \\
\text { (Cycles per Degree) }\end{array}$} \\
\cline { 2 - 4 } \multicolumn{1}{c}{ Relationship } & \multicolumn{1}{c}{1} & 4 & 12 \\
\hline Linear & 98 & 682 & 1023 \\
Quadratic & 97 & 550 & 26 \\
Logarithmic & 231 & 281 & 393 \\
Two-Limb Linear & 43 & 178 & 23 \\
\hline
\end{tabular}


consider the data to show that the approximately constant relationship between response duration and stimulus duration breaks down at the same stimulus duration $(100 \mathrm{msec})$ for each spatial frequency, indicating that each spatial frequency produces the same total response duration. Examination of their Table 1 shows that this conclusion is not strongly supported.' The relationship breaks down between 75 and $100 \mathrm{msec}$ for the 1-cycle/deg grating, between 100 and $150 \mathrm{msec}$ for the 4-cycles/deg grating, and between 150 and $200 \mathrm{msec}$ for the 12-cycles/deg grating. (The SOA for the 12-cycles/deg 150-msec condition is actually $379 \mathrm{msec}$.)

The data thus show an approximately constant SOA for each spatial frequency at short stimulus durations and that the limiting stimulus duration varies from between 75 and $100 \mathrm{msec}$ for $1 \mathrm{cycle} /$ deg to between 150 and $200 \mathrm{msec}$ for 12 cycles/deg. This conclusion is consistent with that reached by Bowling and Lovegrove (1980). The use of either ISI as a measure of persistence or SOA as a measure of response duration therefore indicates that response duration increases with that reached by Bowling and Lovegrove (1980). The use of either ISI as a measure of persistence or SOA as a measure of response duration therefore indicates that response duration increases with increasing spatial frequency. As argued above, SOA is an appropriate measure of response time, but, by definition, is not an appropriate measure of persistence. The conclusion that response time increases with spatial frequency is also consistent with recent data obtained by Di Lollo and Woods (in press).

The 100-msec rule was proposed by Di Lollo (1977) using arrays of light pulses. Using the same procedure, Di Lollo and Woods (in press) have demonstrated that response time is not always $100 \mathrm{msec}$ but varies with spatial frequency (altered by optical defocusing). Their results support the proposal that a constant response duration results from short stimulus presentations, but show that this response duration increases with spatial frequency, as suggested by Bowling and Lovegrove (1980). Further support for this proposal comes from the finding that high spatial frequency gratings have longer critical durations than low-frequency gratings (Breitmeyer \& Ganz,
1977; Legge, 1978). Consequently, the breakdown in the approximate constancy of the SOA at brief stimulus durations may occur at various durations, depending upon spatial frequency. If response duration is determined by the duration of temporal integration, high-frequency gratings would be expected to produce longer response durations than lower frequency gratings. The analysis of the data in terms of SOA basically supports this prediction. The reanalysis by Stanley et al. (1980) of the data of Bowling and Lovegrove (1980) thus does not alter the conclusions of the latter authors relating persistence and critical duration. Finally, it may be slightly misleading to talk in terms of a 100 -msec rule, as this duration applies only to a specific set of stimulus conditions.

\section{REFERENCES}

Bowling, A., \& Lovegrove, W. The effect of stimulus duration on the persistence of gratings. Perception \& Psychophysics, $1980,27,574-568$.

Breitmeyer, B. G., \& Ganz, L. Temporal studies with flashed gratings: Inferences about human transient and sustained channels. Vision Research, 1977, 17, 861-865.

Di Lollo, V. Temporal characteristics of iconic memory. Nature, $1977,267,241-243$.

Di LoLLo, V. Temporal integration in visual memory. Journal of Experimental Psychology: General, 1980, 109, $75-97$.

Di Lollo, V., \& Woods, E. Duration of visible persistence in relation to a range of spatial frequencies. Journal of Experimental Psychology: Human Perception and Performance, in press.

Erron, R. The relationship between the duration of a stimulus and the duration of a perception. Neuropsychologica, 1970, 8, 37-55.

LEGGE, G. E. Sustained and transient mechanisms in human vision: Temporal and spatial properties. Vision Research, 1978, 18, 69-81.

OWEN, W. G. Spatio-temporal integration in the human peripheral retina. Vision Research, 1972, 12, 1011-1026.

Stanley, G., Howell, E. R., \& Sмith, G. Stimulus duration and grating persistence: An instance of the $100-\mathrm{msec}$ rule? Perception \& Psychophysics, 1980, 28, 597-598.

\section{NOTE}

1. Unfortunately, some of the estimates in Table 1 of Stanley et al. are incorrect. In fact, for every spatial frequency, the SOA invariably increases with each increase in duration.

(Received for publication October 13, 1980; accepted October 20, 1980.) 